\title{
ASSOCIATION BETWEEN ANTHROPOMETRIC PARAMETERS, PHYSICAL ACTIVITY LEVEL, GENDER AND PLACE OF RESIDENCE IN PRESCHOOL CHILDREN
}

${ }^{1}$ Sonja Djuric, ${ }^{1}$ Vesna Gluvic-Celic, ${ }^{1}$ Cedomir Oljaca, ${ }^{2}$ Milorad Vujnic

${ }^{1}$ Public Health Institution "Dom zdravlja" Gradiska, BiH

${ }^{2}$ Faculty of medicine, University of Banja Luka, BiH

Obesity in childhood is a global problem, influenced by socio-demographic and cultural factors.

The aim of our research was to determine the correlation of the anthropometric parameters of pre-school children (ages 6 and 7) with the level of physical activity, gender and place of residence.

The research was conducted in February and March 2018. The study involved 200 examinees (102 boys and 98 girls). The values of the observed parameters are obtained by standard procedures of anthropometric measurements. Parents filled in the appropriate questionnaire.

The nutritional status is determined according to the WHO parameters (overweight BMI $\geq 85$ percentile, obesity $\geq 95$ percentile).

$>$ The mean values of body weight, height, BMI and waist circumference showed no statistically significant difference in relation to gender ( $p>0.05)$.

$>$ The percentage distribution of BMI shows that $3 \%$ was underweight, $61.50 \%$ had physiologic nutrition, $13 \%$ were overweight and $22.50 \%$ were obese. All underweighted subjects were male. Out of a total of 45 obese subjects, 27 were male (60\%) and 18 female (40\%).

There was no statistically significant difference in the mean values of the observed parameters relative to the place of residence ( $p>0.05)$.

The results showed that physically less active children have statistically significantly higher values of body weight, BMI and waist circumference compared to physically more active children $(p<0.05)$.

Our results show that physical activity has significantly influences on the nutritional status of children, and therefore it is necessary to develop educational programs of quality physical activity.
Graph 1. Examinees by gender and year of birth

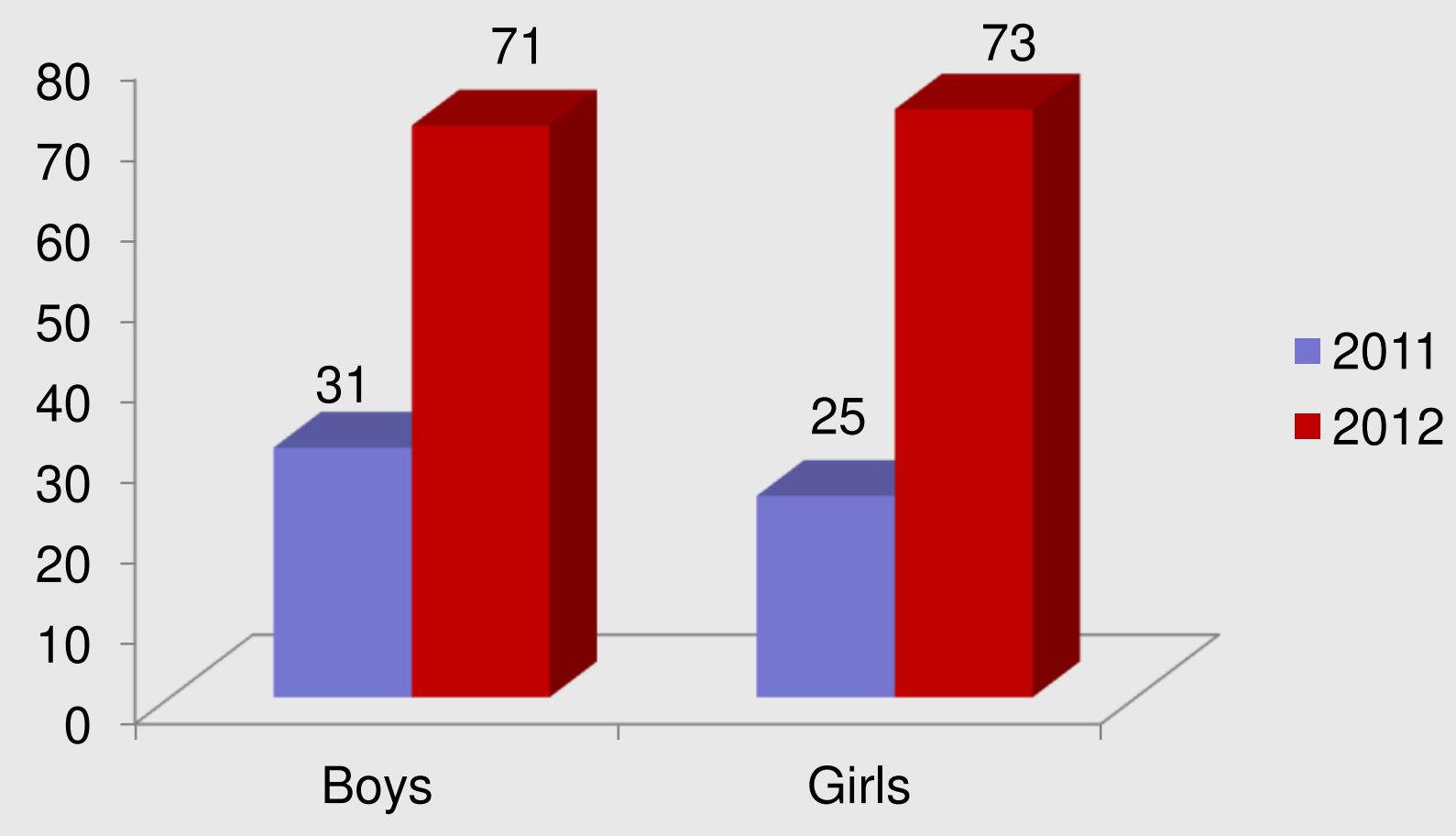

Table 1. Characteristics of the examinees (by gender)

\begin{tabular}{|c|c|c|c|c|c|}
\hline \multirow{2}{*}{ Gender } & \multicolumn{2}{|c}{ Boys } & \multicolumn{2}{c|}{ Girls } & \multirow{2}{*}{ p-value } \\
\cline { 2 - 6 } & Mean & SD & Mean & SD & \\
\hline Body mass $(\mathrm{kg})$ & 23.799 & 4.826 & 23.148 & 4.398 & 0.320 \\
\hline Body height $(\mathrm{cm})$ & 118.832 & 4.896 & 117.755 & 5.628 & 0.149 \\
\hline WC $(\mathrm{cm})$ & 55.333 & 6.705 & 54.7245 & 6.357 & 0.511 \\
\hline BMI & 16.756 & 2.587 & 16.628 & 2.475 & 0.720 \\
\hline
\end{tabular}

Table 2. Characteristics of the examinees (by place of residence)

\begin{tabular}{|c|c|c|c|c|c|}
\hline \multirow{2}{*}{$\begin{array}{c}\text { Place of } \\
\text { residence }\end{array}$} & \multicolumn{2}{|c|}{ City area } & \multicolumn{2}{c|}{ Rural area } & p-vaule \\
\cline { 2 - 6 } & Mean & SD & Mean & SD & \\
\hline Body mass $(\mathrm{kg})$ & 23.796 & 4.151 & 23.325 & 4.843 & 0.477 \\
\hline Body height $(\mathrm{cm})$ & 118.894 & 5.087 & 118.019 & 5.087 & 0.263 \\
\hline WC (cm) & 55.652 & 6.95 & 54.731 & 6.313 & 0.366 \\
\hline BMI & 16.774 & 2.316 & 16.653 & 2.632 & 0.741 \\
\hline
\end{tabular}

Table 3. Characteristics of the examinees (by weekly activity)

\begin{tabular}{|c|c|c|c|c|c|}
\hline \multirow{2}{*}{ Weekly activity } & \multicolumn{4}{|c|}{ Less than 3 days } & \multicolumn{3}{c|}{ More than 3 days } & \multirow{2}{*}{ p-vaule } \\
\cline { 2 - 6 } & Mean & SD & Mean & SD & \\
\hline Body mass $(\mathrm{kg})$ & 27.124 & 3.827 & 23.199 & 4.561 & 0.002 \\
\hline Body height $(\mathrm{cm})$ & 120.179 & 6.556 & 118.167 & 5.168 & 0.28 \\
\hline WC $(\mathrm{cm})$ & 61.286 & 6.925 & 54.565 & 6.267 & 0.003 \\
\hline BMI & 18.899 & 2.652 & 16.527 & 2.445 & 0.006 \\
\hline
\end{tabular}

Table 4. Distribution of BMI (percentile of BMI)

\begin{tabular}{|c|c|c|c|c|c|c|}
\hline \multirow{2}{*}{ BMI } & \multicolumn{2}{|c|}{ Boys } & \multicolumn{2}{c|}{ Girls } & \multicolumn{2}{c|}{ total } \\
\cline { 2 - 8 } & $\mathrm{N}$ & $\%$ & $\mathrm{~N}$ & $\%$ & $\mathrm{~N}$ & $\%$ \\
\hline Underweight & 6 & $5.88 \%$ & 0 & $0.00 \%$ & 6 & $3.00 \%$ \\
\hline Physiologic nutr. & 57 & $55.88 \%$ & 66 & $67.35 \%$ & 123 & $61.50 \%$ \\
\hline Overweight & 12 & $11.76 \%$ & 14 & $14.29 \%$ & 26 & $13.00 \%$ \\
\hline Obese & 27 & $26.47 \%$ & 18 & $18.37 \%$ & 45 & $22.50 \%$ \\
\hline
\end{tabular}
sonja_djuric91@yahoo.com 